\title{
Cosmological results from Planck and LSS
}

\section{François Bouchet}

Institut d'Astrophysique de Paris, CNRS and Sorbonne Universités-UPMC

E-mail: boucheteiap. fr

Sketched out in 1992, selected by ESA in 1996, launched in 2009, Planck delivered on March 21 st its first full sky maps of the millimetric emission at 9 frequencies, as well as those which follow from them, and in particular Planck map of the anisotropies of the Cosmic Microwave Background (CMB). The later displays minuscule variations as a function of the observing direction of the temperature of the fossile radiation around its mean temperature of $2.725 \mathrm{~K}$. I will briefly describe how these high resolution maps with a precision of a few parts in a million have been obtained, from collection to analysis of the first 500 billion samples of our HFI instrument. $\mathrm{CMB}$ anisotropies reveal the imprint of the primordial fluctuations which initiate the growth of the large scale structures of the Universe, as transformed by their evolution, in particular during the first 370000 years, i.e. till the Universe became transparent and the forming of the image we record today. The statistical characteristics of these anisotropies allow constraining jointly the physics of the creation of the primordial fluctuations and that of their evolution. They teach us the possible value of the parameters of the models which we confront to data. I will describe Planck estimates of the density of the constituents of the Universe (usual matter, cold dark matter or CDM, dark energy...), and their implication in terms of derived quantities like the expansion rate or the spatial curvature. I will review what we learnt on the generation of the fluctuation, and wil discuss extensions of the standard cosmological model, so called "Lambda-CDM", both in term of non minimal physical models - multi-field inflation for instance, or additional constituents like cosmic strings or a fourth neutrino.

Finally, it will briefly describe other promising results on the matter distribution which is travelled through by the CMB image on its long 13.7 billion years trip towards us. I will mention in particular what we can learn on the dark matter distribution - which is detected through its distorting effet of the CMB image by gravitationnal lensing, or that of hot gaz, which is revealed by the spectral distortion it induces.

Frontiers of Fundamental Physics 14 - FFP14,

15-18 July 2014

Aix Marseille University (AMU) Saint-Charles Campus, Marseille

\footnotetext{
* Speaker.
} 